\title{
(2) OPEN ACCESS \\ Efficacy and safety of PD-1/PD-L1 plus CTLA-4 antibodies \pm other therapies in lung cancer: a systematic review and meta-analysis
}

\author{
Xiang Shen, ${ }^{1}$ Shangke Huang, ${ }^{2}$ Hua Xiao, ${ }^{1}$ Shan Zeng, ${ }^{1}$ Jiexing Liu, Zhuolan Ran, \\ Bin Xiong'
}

\begin{abstract}
- Additional supplemental material is published online only. To view, please visit the journal online (http://dx. doi.org/10.1136/ejhpharm2021-002803)
\end{abstract}

'Department of Respiratory and Critical Care Medicine, The Affiliated Hospital of Southwest Medical University, Luzhou, Sichuan, China Department of Oncology, The Affiliated Hospital of Southwest Medical University, Luzhou, Sichuan, People's Republic of China

Correspondence to Dr Bin Xiong, Department of Respiratory and Critical Care Medicine, The Affiliated University, Luzhou, China; xionbin321@163.com

$\mathrm{XS}$ and SH contributed equally.

$\mathrm{XS}$ and $\mathrm{SH}$ are joint first authors.

Received 22 March 2021 Accepted 24 August 2021

EAHP Statement 4: Clinical Pharmacy Services. EAHP Statement 5: Patient Safety and Quality Assurance. Hospital of Southwest Medical

\section{ABSTRACT \\ Purpose To investigate the efficacy and safety of programmed cell death 1 (PD-1)/programmed cell death- ligand 1 (PD-L1) plus cytotoxic T lymphocyte antigen-4 (CTLA-4) antibodies \pm other therapies in patients with advanced lung cancer.}

Methods In accordance with the retrieval strategy, we searched electronic databases for randomised controlled trials testing PD-1/PD-L1 plus CTLA-4 antibodies in patients with lung cancer; RR (for objective response rate (ORR), overall survival (OS), progression-free survival (PFS), and immune-related adverse events (irAEs)) from individual studies were calculated and pooled by using random-effects models or fixed-effects models; heterogeneity and publication bias analyses were also performed, using Review Manager 5.3 and Stata 15.1 for statistical analysis.

Results We included six studies. Four different immune checkpoint inhibitors (nivolumab, pembrolizumab, durvalumab, tremelimumab) were used. Dual checkpoint inhibitors \pm other therapies for advanced lung cancer showed significant improvements in ORR (RR 1.49, $95 \% \mathrm{Cl} 1.11$ to 1.98; $p=0.007)$, OS (HR $0.72,95 \% \mathrm{Cl}$ 0.63 to $0.83 ; p<0.00001)$, and PFS (HR $0.72,95 \% \mathrm{Cl}$ 0.63 to $0.82 ; p<0.00001)$. The subgroup analyses were consistent with the pooled results. The PD-L1 $\geq 1 \%$ (HR 0.67, 95\% Cl 0.54 to 0.82; $\mathrm{p}<0.0001$ ) subgroup differences indicated a statistically significant subgroup effect, but the PD-L1 <1\% subgroup (HR 0.88, 95\% Cl 0.75 to $1.05 ; p=0.15$ ) was not statistically significant. The incidence of adverse events (grade $\geq 3$ ) was lower than that of the control group (RR $0.90,95 \% \mathrm{Cl} 0.80$ to $1.02 ; p=0.09)$, but was not significant.

Conclusions PD-1/PD-L1 inhibitors combined with CTLA-4 inhibitors \pm other therapies can improve the ORR, OS and PFS of patients with advanced or metastatic lung cancer, but the incidence of adverse reactions is high although generally tolerable.

PROSPERO registration CRD42020149216.

Check for updates

(c) European Association of Hospital Pharmacists 2021. Re-use permitted under CC BY-NC. No commercial re-use. Published by BMJ.

To cite: Shen $X$, Huang $S$, Xiao $\mathrm{H}$, et al.

Eur J Hosp Pharm Epub ahead of print: [please include Day Month Year]. doi:10.1136/

ejhpharm-2021-002803

\section{INTRODUCTION}

Lung cancer is the most deadly cancer; the 5 year survival rate of patients with stage IV is only $4 \% .{ }^{1}$ We need to actively find other therapies to resolve this dilemma. Studies have found that cancer cells in the context of the tumour microenvironment (TME) through regulatory $\mathrm{T}$ cells (Tregs) change immune homeostasis, suppressing the activation and effector functions of the innate and acquired immune systems, and thereby escape immune surveillance. ${ }^{23}$ Immune checkpoint inhibitors (ICIs) restore and maintain the function of the immune system against tumour cells by blocking specific signalling pathways, in which cytotoxic $\mathrm{T}$ cells play an important role in immune monitoring and antitumour responses. ${ }^{4}$ Compared with chemotherapy, programmed cell death 1 (PD-1)/programmed cell death-ligand 1 (PD-L1) plus cytotoxic T lymphocyte antigen-4 (CTLA-4) inhibitors have higher objective response rates, lead to longer overall survival, and have a better toxicity profile. ${ }^{5}{ }^{6}$ In particular, patients without targeted cancer mutation genes receiving tumour immunotherapy are showing improvements in their long-term survival rates, ${ }^{78}$ and it is the new treatment standard for patients with newly diagnosed advanced non-small cell lung cancer (NSCLC) who are in general good condition and have no contraindications. ${ }^{9}$

However, mono-immunotherapy only significantly prolongs survival in patients with high PD-L1, ${ }^{10}$ which is only beneficial to a small number of patients. ${ }^{11}$ Greater therapeutic effectiveness can be achieved at the cost of increased side effects through synergistic or combined treatment. Recent clinical trials have demonstrated that the dual checkpoint inhibitors ipilimumab plus nivolumab can synergistically enhance $\mathrm{T}$ cell function and the immune system's ability to fight tumour cells through different and complementary mechanisms, ${ }^{12}$ and have a better curative effect than nivolumab monotherapy. The effect was better in patients with high PD-L1, ${ }^{13}$ and it reduced the proportion of patients with advanced tumours. ${ }^{14}$

Immune-related adverse events (irAEs), which are different from chemotherapy-related toxic reactions, ${ }^{15}$ are caused by upregulated immune systems leading to severe inflammatory reactions and immune toxicity. ${ }^{16}$ Some trials have observed serious adverse reactions and have been stopped. ${ }^{17}$

Several clinical trials have been conducted to evaluate the efficacy and toxicity of PD-1/PD-L1 combined with CTLA-4 antibodies in patients with advanced lung cancer, but the results such as progression-free survival (PFS) and overall survival (OS) are controversial. Therefore, we conducted a systematic meta-analysis using data from the published literature to evaluate the efficacy and safety of PD-1/PD-L1 combined with CTLA-4 antibody and/or other therapeutic regimens for treating lung cancer. 


\section{MATERIALS AND METHODS}

We followed the principles of the systemic review and metaanalysis (PRISMA) guidelines.

\section{Systematic review and meta-analysis of all RCTs testing PD-1/ PD-L1 plus CTLA-4 antibodies \pm other therapies for lung cancer}

Data sources and search strategy

We searched PubMed, Web of Science, the Cochrane Library, Ovid, and Embase for randomised controlled trials (RCTs) testing PD-1/PD-L1 plus CTLA-4 antibodies in patients with lung cancer, without any date restrictions. The following retrieval strategy was employed: (PD-1 OR PD-L1 OR Pembrolizumab OR Nivolumab OR Cemiplimab-rwlc OR Toripalimab OR Sintilimab OR Atezolizumab OR Avelumab OR Durvalumab) AND (CTLA-4 OR Ipilimumab OR Tremelimumab) AND lung cancer AND trial. Manual searches were also performed for additional references that met the inclusion criteria to avoid missing any studies.

\section{Inclusion and exclusion criteria}

Inclusion and exclusion criteria were independently evaluated by two researchers based on the PICO principle. The included studies had to meet the following criteria: (1) studies published in English; (2) randomised controlled clinical trials to study the efficacy and safety of (PD-1 OR PD-L1 OR Pembrolizumab OR Nivolumab OR Cemiplimab-rwlc OR Toripalimab OR Sintilimab OR Atezolizumab OR Avelumab OR Durvalumab) AND (CTLA-4 OR Ipilimumab OR Tremelimumab); (3) the patients were histologically diagnosed with lung cancer, not including HIV, organ transplantation, viral infection and other special patients; (4) presents analysis-related data of objective response rate (ORR), PFS, OS, and toxicity. The following studies were excluded: (1) reviews, editorials, case reports, or animal and cellular studies; (2) required data are incomplete; (3) no dose and usage information.

\section{Data extraction and quality assessment}

Two researchers independently conducted the study selection process based on the inclusion and exclusion criteria, and extracted the following information: first author name, publication year, trial phase, clinical trial number, trial design, pathological type, sample size, usage and dosage, follow-up time, median OS and PFS, and irAEs. If there were disagreements in the study selection and data extraction process, a consensus was reached through discussion or when necessary a third investigator was consulted. We used the method of Jadad et $a l^{18}$ to evaluate the quality of the included studies.

\section{Data synthesis and statistical analysis}

We used Review Manager 5.3 and Stata 15.1 for statistical analysis. The $\mathrm{Q}$ test and $\mathrm{I}^{2}$ statistics were used to evaluate the statistical heterogeneity. When $\mathrm{p}<0.05$ or $\mathrm{I}^{2}>50 \%$, heterogeneity was statistically significant, and we used random-effects models; otherwise, we used fixed-effect models. HRs $>1$ of OS and PFS indicated it was beneficial to the control group, while HRs $<1$ indicated it was beneficial to the PD-1/PD-L1 combined CTLA-4 antibody group. ORs $>1$ of ORR and adverse events (AEs) means a higher effective rate and toxicity, while ORs $<1$ indicates a lower effective rate and safety. $\mathrm{P}<0.05$ was considered statistically significant, and all $\mathrm{p}$ values were bilateral.

\section{RESULTS}

\section{Study characteristics and risk of bias}

Online supplemental figure 1 shows a flow chart of the study. A preliminary literature search screened a total of 3983 records from the databases: 3317 of them were left after deleting duplicate records; 86 were left after screening; 43 were left after screening the titles and abstracts; and six studies were left after reviewing each publication in full. All relevant references have been reviewed. Finally, six RCTs were included in this metaanalysis (one of the trials employed two trials to compare the efficacy and toxicity with the control group, which were conducted as two independent trials) (online supplemental figure 1), all using the response evaluation standard (RECIST) or WHO standards.

All of the trials were randomised, controlled, open-label clinical trials. They included 952 experimental patients and 869 control patients, ${ }^{19-23}$ and mainly studied ipilimumab + nivolumaband durvalumab +tremelimumab for the treatment of lung cancer. Online supplemental table 1 summarises the detailed characteristics of the included studies.

We assessed the quality of each study in this analysis based on the Jadad score, and online supplemental figure 2 provides the risk results of the bias assessment.

\section{Overall response rate}

Finally, six studies conducted on 952 experimental-arm patients and 896 control-arm patients met the inclusion criteria and were included in the ORR analysis. The ORR funnel plot shows significant asymmetry (online supplemental figure 3), and heterogeneity among the studies was significant $\left(\mathrm{p}=0.02, \mathrm{I}^{2}=61 \%\right)$. The pooled RR for ORR was calculated with a random-effects model. This meta-analysis showed that ORR was significantly improved in the treatment of PD-1/PD-L1 plus CTLA-4 antibodies \pm other therapies (RR $1.49,95 \%$ CI 1.11 to $1.98 ; p=0.007$ ) (figure 1 ), which was statistically significant. The clinical efficacy of PD-1/

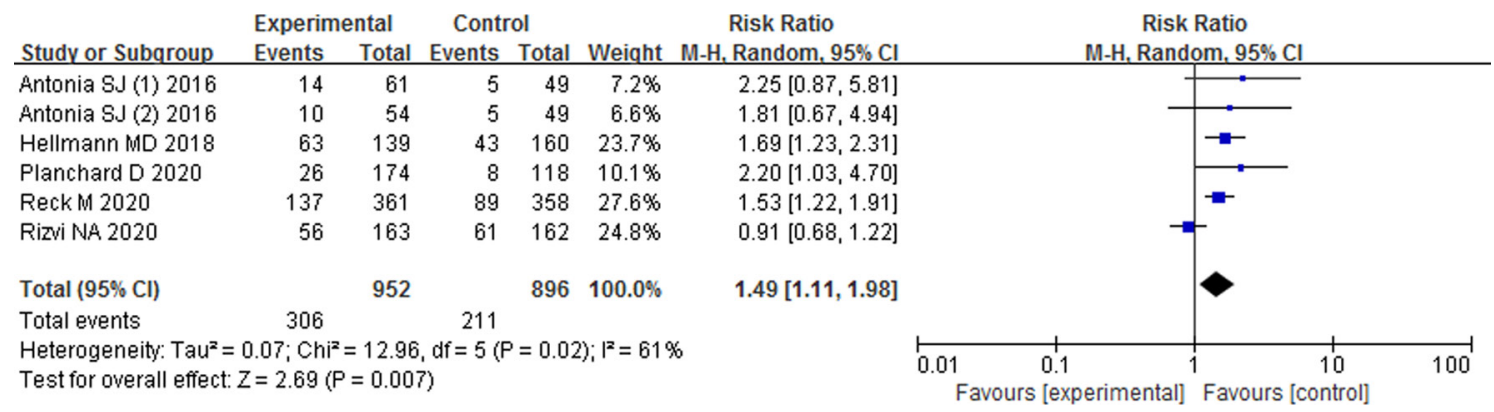

Figure 1 Forest plot of ratio risk and 95\% confidence intervals for overall response rate of PD-1/PD-L1 plus CTLA-4 antibodies with lung cancer. CTLA-4, cytotoxic T lymphocyte antigen-4; PD-1, programmed cell death 1; PD-L1, programmed cell death-ligand 1. 


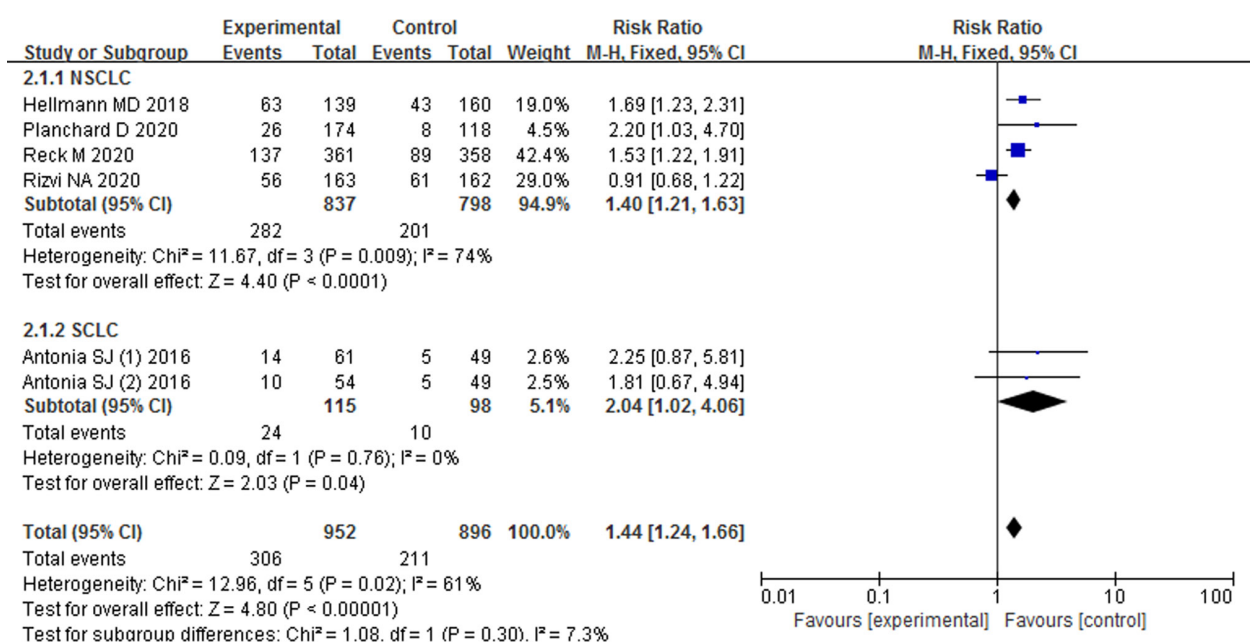

Figure 2 Forest plot of ratio risk for overall response rate in the non-small cell lung cancer (NSCLC) and small cell lung cancer (SCLC) subgroups in patients who received PD-1/PD-L1 plus CTLA-4 antibodies. CTLA-4, cytotoxic T lymphocyte antigen-4; PD-1, programmed cell death 1; PD-L1, programmed cell death-ligand 1.

PD-L1 combined with CTLA-4 antibodies \pm other therapies in patients with advanced lung cancer was higher than that in the control group. In subgroup analysis (figure 2), regardless of whether it is NSCLC (RR $1.40,95 \%$ CI 1.21 to $1.63 ; \mathrm{p}<0.0001)$ or small cell lung cancer (SCLC) (RR 2.04, 95\% CI 1.02 to 4.06; $\mathrm{p}=0.04)$, the results showed that dual ICI therapies \pm other therapies improved ORR. The meta-analysis results did not change significantly throughout the sensitivity analysis, indicating that the sensitivity is low and the result is robust and credible.

\section{Overall survival}

Three trials reported OS combined HRs and 95\% CI, and heterogeneity among the studies was not significant $\left(\mathrm{p}=0.3, \mathrm{I}^{2}=17 \%\right)$. The results showed that the OS of patients treated with PD-1/ PD-L1 combined with CTLA-4 antibodies \pm other therapies was improved (HR 0.72 , 95\% CI 0.63 to 0.83 ; p < 0.00001 ) (figure 3).

In subgroup analysis (figure 4), PD-L1 $\geq 1 \%$ (HR 0.67, 95\% CI 0.54 to $0.82 ; \mathrm{p}<0.0001)$ subgroup differences indicated a statistically significant subgroup effect, but the PD-L1 $<1 \%$ subgroup (HR $0.88,95 \%$ CI 0.75 to $1.05 ; \mathrm{p}=0.15$ ) was not statistically significant (figure 4). This means that lung cancer patients with PD-L1 expression $\geq 1$ benefit more from dual immunotherapy \pm other therapies.

\section{Progression-free survival}

Four trials were included for PFS evaluation. The funnel plot of PFS shows symmetry, and heterogeneity among the studies was not significant $\left(\mathrm{p}=0.11, \mathrm{I}^{2}=50 \%\right)$ (figure 5$)$. The pooled HR for PFS was calculated using a fixed-effects model. In the treatment of lung cancer with PD-1/PD-L1 combined with CTLA-4 antibodies \pm other therapies, the HR of PFS improved statistically (HR $0.72,95 \%$ CI 0.63 to $0.82 ; \mathrm{p}<0.00001$ ) (figure 5 ).

\section{Immune-related adverse events}

There were five studies with data about severe AEs (grade $\geq 3$ ) and no heterogeneity $\left(\mathrm{p}=0.22, \mathrm{I}^{2}=30 \%\right)$. The pooled RR for AEs (grade $\geq 3$ ) was conducted with a fixed-effects model. The incidence of AEs (grade $\geq 3$ ) was lower than the control group (RR $0.90,95 \%$ CI 0.80 to $1.02 ; p=0.09$ ) (figure 6), but it was not significant. Among them, rash and diarrhoea were the most common, and anaemia and neutrophilic granulocyte counts were the most common during chemotherapy (figure 6).

\section{Publication bias}

Funnel plots and Egger's test were applied to assess the publication bias. For the impact of ORR, the funnel plots were not basically symmetrical, suggesting a high risk of potential publication bias in these studies. For the impact of RR on ORR, Begg's test $(Z=0.00, p=1)$ (online supplemental figure 4) and Egger's test $(\mathrm{T}=0.81, \mathrm{p}=0.462)$ (online supplemental figure 5 ) verified low publication bias. There was no significant publication bias in these studies (online supplemental figures 4 and 5).

\section{DISCUSSION}

Most lung cancer patients receive chemotherapy and/or radiation therapy, ${ }^{1}$ which have poor anti-tumour effects. In recent years, some clinical trials have achieved satisfactory results in the treatment of lung cancer with PD-1/PD-L1 and CTLA-4 antibodies, among which pembrolizumab and nivolumab can prolong the survival of patients with advanced NSCLC, ${ }^{24} 25$

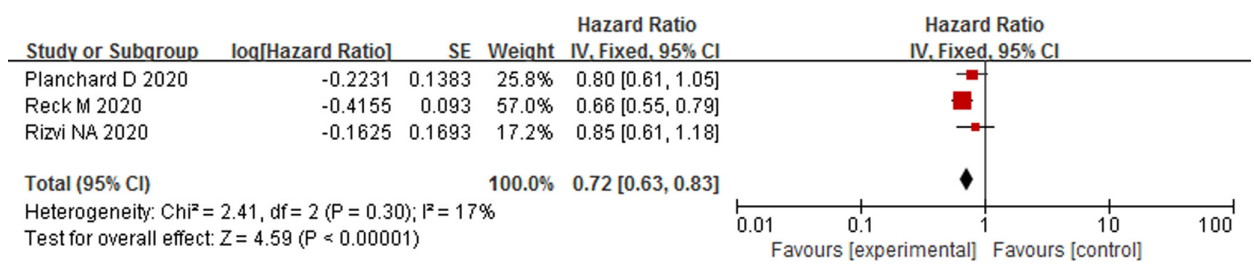

Figure 3 Forest plot of hazard ratios and 95\% confidence intervals for overall survival of PD-1/PD-L1 plus CTLA-4 antibodies with lung cancer. CTLA-4, cytotoxic T lymphocyte antigen-4; PD-1, programmed cell death 1; PD-L1, programmed cell death-ligand 1. 


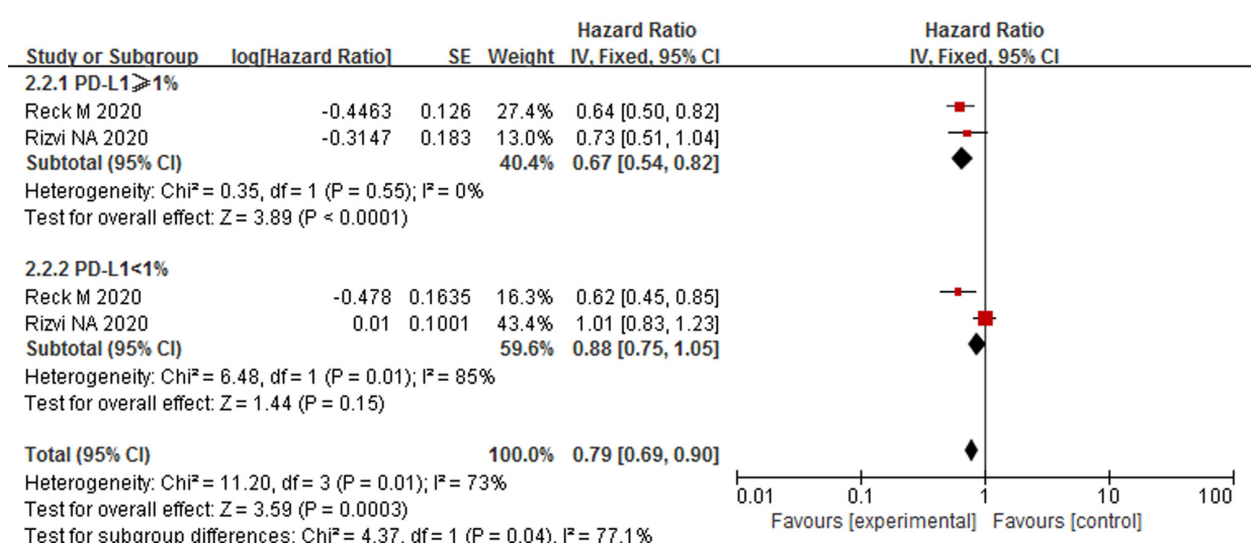

Figure 4 Forest plot of hazard ratios for overall survival in the PD-L1 $\geq 1 \%$ and PD-L1 $<1 \%$ subgroups in patients who received PD-1/PD-L1 plus CTLA-4 antibodies. CTLA-4, cytotoxic T lymphocyte antigen-4; PD-1, programmed cell death 1; PD-L1, programmed cell death-ligand 1.

and durvalumab combined with chemotherapy can effectively improve the prognosis of patients with extensive-stage SCLC (ES-SCLC). ${ }^{26}$ This meta-analysis investigated the efficacy of PD-1/PD-L1 combined with CTLA-4 inhibitors \pm other therapies for lung cancer patients.

$\mathrm{T}$ cells play an important role in the anti-tumour effect. However, $<10 \%$ of CD $8+\mathrm{T}$ cells in tumours have the ability to recognise cancer cells, ${ }^{27}$ and the anti-cancer effect may be caused by $\mathrm{T}$ cells entering the tumour from the peripheral blood after treatment. ${ }^{28}$ Therefore, it is particularly important to increase the number of $\mathrm{T}$ cells and enhance the effector function through different mechanisms by combining PD-1/PD-L1 and CTLA-4 inhibitors. The combined blocking of the CTLA-4 and PD-1/PD-L1 pathways increases the ratio of Teff to Tregs and myeloid-derived suppressor cells (which significantly suppresses the ability of cells to respond), thereby reducing immunosuppression and promoting inflammation in the TME. ${ }^{29}$ At the same time, anti-CTLA-4 antibodies have antibody-dependent cell-mediated cytotoxicity, and they reduce the negative effect of PD-1 antibodies on activating Tregs; CTLA-4 antibodies lead to compensatory overexpression of tumour PD-L1, and PD-1 antibodies block the binding of PD-L1 to PD-1. CTLA-4 antibodies also increase the number of activated $\mathrm{T}$ cells and $\mathrm{Tm}$ in the peripheral blood, and PD-1 antibodies relieve the inhibition of $\mathrm{T}$ cell anti-cancer activity by tumours. Our results revealed the significant effect of dual checkpoint inhibitor treatment for advanced lung cancer, showing significant improvements in ORR (RR $1.49,95 \%$ CI 1.11 to $1.98 ; \mathrm{p}=0.007$ ), OS (HR 0.72, $95 \%$ CI 0.63 to $0.83 ; \mathrm{p}<0.00001$ ), and PFS (HR $0.72,95 \% \mathrm{CI}$ 0.63 to $0.82 ; \mathrm{p}<0.00001)$.

We found that a combination of immunosuppressants and chemotherapy had better anti-tumour effects. By activating AKT, cisplatin rapidly increases the expression of PD-L1 in the TME in a dose-dependent manner, increasing the clinical efficacy of immunotherapy ${ }^{3031}$; it does not affect the immune background of NSCLC after induction, nor does it significantly damage the anti-tumour immune response, ${ }^{32}$ but its effects may not persist.

In addition, when given concurrently, radiotherapy can restore the recruitment of Th1 lymphocytes in the TME, ${ }^{33}$ stimulate antigen presentation, increase tumour antigenicity, ${ }^{34}$ form an 'immune centre', and promote the tumour immune response. $^{3536}$ Through more effective control of the immune system, local therapy is converted into systemic therapy, which results in a synergistic and more effective activity against tumour cells. $^{37}$

Regarding when to use combination therapy, a meta-analysis by Zhou $e a^{38}$ suggests that patients with large tumour volumes should be treated with immunotherapy combined with other therapies to produce stronger and more long-term efficacy, while patients with small tumour volumes or extremely high PD-L1 tumour proportion score should be treated with immunomonotherapy alone. ${ }^{39}$ However, this requires more clinical trials for validation. For lung cancer patients with negative expression of PD-L1, tumour antigens can be released during a short period of chemotherapy at the early stage of treatment. At this time, by activating the patient's own immune system using double immune therapy, it not only enhanced the immune treatment effect, but also avoided the accumulation of long-term chemotherapy side effects. However, regardless of PD-L1 expression level, a high tumour mutational burden (TMB) of NSCLC patients, PD-L/PD-L1 plus CTLA-4 inhibitors had significantly longer PFS than chemotherapy. ${ }^{20}$ Further trials are also needed to explore the selection of immunotherapy therapies based on biomarkers.

The related adverse reactions of PD-1/PD-L1 combined with CTLA-4 inhibitors cannot be ignored. The mechanism may be related to Tregs. Tregs are one of the most abundant suppressor cells in the TME, and they express a large number of checkpoint molecules such as CTLA-4 and PD-1 to maintain immune homeostasis and avoid autoimmunity. Therefore, the targeting

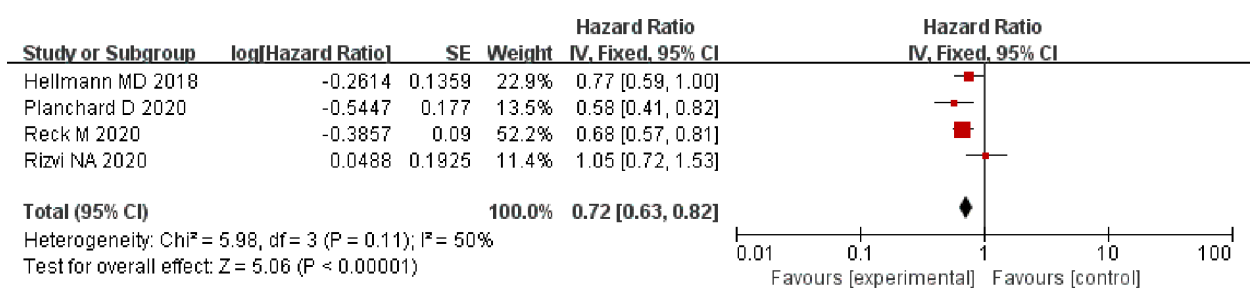

Figure 5 Forest plot of hazard ratios and 95\% confidence intervals for progression-free survival of PD-1/PD-L1 plus CTLA-4 antibodies with lung cancer. CTLA-4, cytotoxic T lymphocyte antigen-4; PD-1, programmed cell death 1; PD-L1, programmed cell death-ligand 1. 


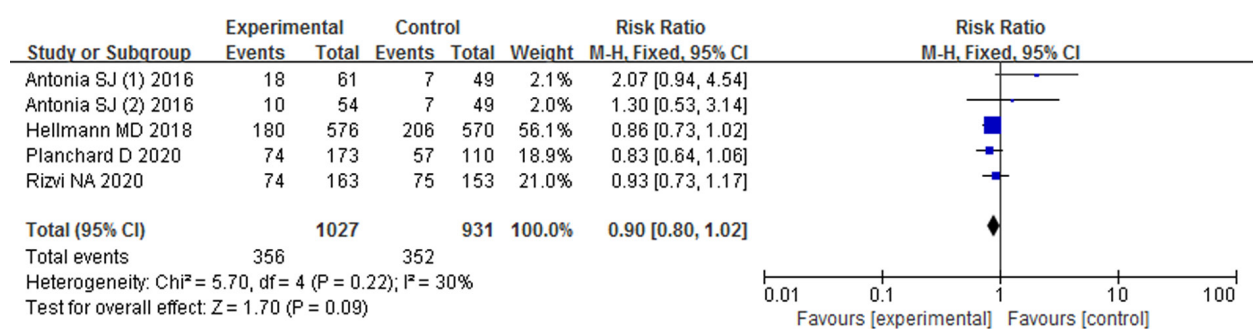

Figure 6 Forest plot of ratio risk and 95\% confidence intervals for immune-related adverse events of PD-1/PD-L1 plus CTLA-4 antibodies with lung cancer. CTLA-4, cytotoxic T lymphocyte antigen-4; PD-1, programmed cell death 1; PD-L1, programmed cell death-ligand 1.

effect of ICIs on Tregs may lead to the occurrence of irAEs. ${ }^{40}$ These events are usually controllable ${ }^{41}$ and can be effectively controlled and managed by immunosuppressive methods such as steroids. ${ }^{42}$ However, there are still a few patients who have serious adverse reactions, with the highest mortality caused by neurological and cardiac toxicity. ${ }^{43}$ The risk of irAEs in patients treated with CTLA-4 is dose-related, ${ }^{44}$ and most grade 3 or higher irAEs occur within 8-12 weeks after starting treatment.

PD-1 antibody-related irAEs are relatively low in frequency, and most irAEs occur within the first 6 months of treatment and take longer to manifest than CTLA-4 related toxicity. ${ }^{45}$ The organ involvement spectrum of the two is also different. AntiPD-1 drugs cause arthritis more frequently, and anti-CTLA-4 drugs are more related to colitis. ${ }^{46}$ Combining anti-CTLA-4 antibodies and anti-PD-1 antibodies can increase the incidence and severity of irAEs and cause them to occur earlier. ${ }^{47}$ This is consistent with our research findings.

A large data analysis showed that the risk of immune events is associated with TMB. Cancers with a high TMB, such as NSCLC, are associated with more irAEs during anti-PD-1 therapy. ${ }^{48}$ Receiving low-dose glucocorticoids does not inhibit the immune response caused by ICIs, and patients can continue to maintain a beneficial response to ICIs. ${ }^{44}$ The combination of anti-PD-1 antibodies and immune antibodies of selectively targeted specific inflammatory mediators can prevent or delay the progression of advanced tumours in patients with autoimmune diseases without affecting the anti-tumour effect of the anti-PD-1 antibodies. ${ }^{49}$ It was observed that even if patients receiving PD-1 antibodies stopped treatment due to the reaction, they could have a longer duration of action without the need to rush to the next treatment. ${ }^{50}$ However, long-term exposure to immunosuppressants can lead to rare fatal immune-related events. ${ }^{5152}$

In this meta-analysis, our results showed that the combination of PD-1/PD-L1 and CTLA-4 antibody was effective for the treatment of lung cancer and the occurrence of adverse reactions was controllable (figures 1, 2 and 6). This provides a good option for the treatment of SCLC. A non-randomised controlled trial of ipilimumab and nivolumab given postoperatively to patients with ES-SCLC resulted in better 1 year OS than the control group. ${ }^{53}$ More randomised, multicentre, and large sample studies are needed to confirm and evaluate the cost of these treatment strategies and the quality of life of patients.

Our research also has some limitations that may have affected the final results. First, most of the studies have a small sample size; currently there are very few data about the use of dualimmune checkpoint inhibitor combination therapy, and few studies have applied randomised or blinded methods. Second, we extracted trial data from published articles but we did not have access to the patients' original data, which could lead to biases in the data analysis. Therefore, more large-scale clinical trials are needed to further verify the effectiveness and safety of dual immunosuppressive anti-tumour therapy.

\section{CONCLUSION}

PD-1/PD-L1 inhibitors combined with CTLA-4 inhibitors can improve the ORR and PFS of patients with advanced or metastatic lung cancer, but the incidence of adverse reactions is high, although generally tolerable. The survival of patients with SCLC is shorter than that of patients with NSCLC, ${ }^{2}$ and treatment options are limited. Dual immunosuppressants also have therapeutic effects in SCLC. Despite some limitations, our research indicates that the combination of PD-1/PD-L1 plus CTLA-4 inhibitors may be a promising treatment strategy for patients with advanced lung cancer, but attention should be paid to the occurrence of adverse reactions.

Correction notice This article has been updated since it was published online. The affiliations have been modified.

Contributors None.

Funding This project is supported by the Doctoral Startup Fund of the Affiliated Hospital of Southwest Medical University (no.826).

Competing interests None declared.

Patient consent for publication Not required.

Provenance and peer review Not commissioned; externally peer reviewed.

Data availability statement Data are available in a public, open access repository.

Supplemental material This content has been supplied by the author(s). It has not been vetted by BMJ Publishing Group Limited (BMJ) and may not have been peer-reviewed. Any opinions or recommendations discussed are solely those of the author(s) and are not endorsed by BMJ. BMJ disclaims all liability and responsibility arising from any reliance placed on the content. Where the content includes any translated material, BMJ does not warrant the accuracy and reliability of the translations (including but not limited to local regulations, clinical guidelines, terminology, drug names and drug dosages), and is not responsible for any error and/or omissions arising from translation and adaptation or otherwise.

Open access This is an open access article distributed in accordance with the Creative Commons Attribution Non Commercial (CC BY-NC 4.0) license, which permits others to distribute, remix, adapt, build upon this work non-commercially, and license their derivative works on different terms, provided the original work is properly cited, an indication of whether changes were made, and the use is noncommercial. See: http://creativecommons.org/licenses/by-nc/4.0/.

\section{REFERENCES}

1 Miller KD, Nogueira L, Mariotto $A B$, et al. Cancer treatment and survivorship statistics, 2019. CA Cancer J Clin 2019;69:363-85.

2 Najafi M, Farhood B, Mortezaee K. Contribution of regulatory T cells to cancer: a review. J Cell Physiol 2019;234:7983-93.

3 Vinay DS, Ryan EP, Pawelec G, et al. Immune evasion in cancer: mechanistic basis and therapeutic strategies. Semin Cancer Biol 2015;35 Suppl:S185-98.

$4 \mathrm{Kim} \mathrm{DH}$, Kim H, Choi YJ, et al. Exosomal PD-L1 promotes tumor growth through immune escape in non-small cell lung cancer. Exp Mol Med 2019;51:1-13. 
5 Rittmeyer A, Barlesi F, Waterkamp D, et al. Atezolizumab versus docetaxel in patients with previously treated non-small-cell lung cancer (OAK): a phase 3, open-label, multicentre randomised controlled trial. Lancet 2017;389:255-65.

6 Herbst RS, Baas P, Kim D-W, et al. Pembrolizumab versus docetaxel for previously treated, PD-L1-positive, advanced non-small-cell lung cancer (KEYNOTE-010): a randomised controlled trial. Lancet 2016;387:1540-50.

7 Planchard D, Popat S, Kerr K, et al. Metastatic non-small cell lung cancer: ESMO Clinical Practice Guidelines for diagnosis, treatment and follow-up". Ann Oncol 2019;30:863-70.

8 Schuler M, Paz-Ares L, Sequist LV, et al. First-line afatinib for advanced EGFRm+ NSCLC: analysis of long-term responders in the LUX-Lung 3, 6, and 7 trials. Lung Cancer 2019;133:10-19.

9 Ackermann CJ, Reck M, Paz-Ares L, et al. First-line immune checkpoint blockade for advanced non-small-cell lung cancer: travelling at the speed of light. Lung Cancer 2019;134:245-53.

10 Proto C, Ferrara R, Signorelli D, et al. Choosing wisely first line immunotherapy in nonsmall cell lung cancer (NSCLC): what to add and what to leave out. Cancer Treat Rev 2019;75:39-51.

11 Verma V, Sharma G, Singh A. Immunotherapy in extensive small cell lung cancer. Exp Hematol Oncol 2019;8:5

12 Rizvi NA, Chul Cho B, Reinmuth N, et al. Durvalumab with or without tremelimumab vs platinum-based chemotherapy as first-line treatment for metastatic non-small cell lung cancer: MYSTIC. Ann Oncol 2018;29:x40-1.

13 Hellmann MD, Rizvi NA, Goldman JW, et al. Nivolumab plus ipilimumab as first-line treatment for advanced non-small-cell lung cancer (CheckMate 012): results of an open-label, phase 1, multicohort study. Lancet Oncol 2017;18:31-41.

14 Larkin J, Hodi FS, Wolchok JD. Combined nivolumab and ipilimumab or monotherapy in untreated melanoma. N Engl J Med 2015;373:23-34.

15 Postow MA, Sidlow R, Hellmann MD. Immune-related adverse events associated with immune checkpoint blockade. N Engl J Med 2018;378:158-68.

16 Naqash AR, Appah E, Yang LV, et al. Isolated neutropenia as a rare but serious adverse event secondary to immune checkpoint inhibition. J Immunother Cancer 2019;7:169.

17 Ready N, Hellmann MD, Awad MM, et al. First-Line nivolumab plus ipilimumab in advanced non-small-cell lung cancer (CheckMate 568): outcomes by programmed death ligand 1 and tumor mutational burden as biomarkers. J Clin Oncol 2019;37:992-1000.

18 Jadad AR, Moore RA, Carroll D, et al. Assessing the quality of reports of randomized clinical trials: is blinding necessary? Control Clin Trials 1996;17:1-12.

19 Planchard D, Reinmuth N, Orlov S, et al. Arctic: durvalumab with or without tremelimumab as third-line or later treatment of metastatic non-small-cell lung cancer. Ann Oncol 2020;31:609-18.

20 Hellmann MD, Ciuleanu T-E, Pluzanski A, et al. Nivolumab plus ipilimumab in lung cancer with a high tumor mutational burden. N Engl J Med 2018;378:2093-104.

21 Rizvi NA, Cho BC, Reinmuth N, et al. Durvalumab with or without tremelimumab vs standard chemotherapy in first-line treatment of metastatic non-small cell lung cancer. JAMA Oncol 2020:6:661.

22 Reck M, Ciuleanu T-E, Dols MC, et al. Nivolumab (NIVO) + ipilimumab (IPI) + 2 cycles of platinum-doublet chemotherapy (chemo) vs 4 cycles chemo as first-line (1L) treatment (tx) for stage IV/recurrent non-small cell lung cancer (NSCLC): CheckMate 9LA. J Clin Oncol 2020;38:9501.

23 Antonia SJ, López-Martin JA, Bendell J, et al. Nivolumab alone and nivolumab plus ipilimumab in recurrent small-cell lung cancer (CheckMate 032): a multicentre, openlabel, phase 1/2 trial. Lancet Oncol 2016;17:883-95.

24 Herbst RS, Baas P, Kim D-W, et al. Pembrolizumab versus docetaxel for previously treated, PD-L1-positive, advanced non-small-cell lung cancer (KEYNOTE-010): a randomised controlled trial. Lancet 2016;387:1540-50

25 Borghaei $\mathrm{H}$, Paz-Ares L, Horn L, et al. Nivolumab versus docetaxel in advanced nonsquamous non-small-cell lung cancer. N Engl J Med 2015;373:1627-39.

26 Paz-Ares L, Dvorkin M, Chen Y, et al. Durvalumab plus platinum-etoposide versus platinum-etoposide in first-line treatment of extensive-stage small-cell lung cancer (Caspian): a randomised, controlled, open-label, phase 3 trial. Lancet 2019:394:1929-39.

27 Scheper W, Kelderman S, Fanchi LF, et al. Low and variable tumor reactivity of the intratumoral TCR repertoire in human cancers. Nat Med 2019;25:89-94.

28 Li J, Byrne KT, Yan F, et al. Tumor cell-intrinsic factors underlie heterogeneity of immune cell infiltration and response to immunotherapy. Immunity 2018;49:178-93.

29 Chae YK, Arya A, lams W, et al. Current landscape and future of dual anti-CTLA4 and PD-1/PD-L1 blockade immunotherapy in cancer; lessons learned from clinica trials with melanoma and non-small cell lung cancer (NSCLC). J Immunother Cancer 2018;6:39.

30 Tran L, Allen CT, Xiao R, et al. Cisplatin alters antitumor immunity and synergizes with PD-1/PD-L1 inhibition in head and neck squamous cell carcinoma. Cancer Immunol Res 2017;5:1141-51

31 Fournel L, Wu Z, Stadler N, et al. Cisplatin increases PD-L1 expression and optimizes immune check-point blockade in non-small cell lung cancer. Cancer Lett 2019:464:5-14.

32 Remark R, Lupo A, Alifano M, et al. Immune contexture and histological response after neoadjuvant chemotherapy predict clinical outcome of lung cancer patients. Oncoimmunology 2016;5:e1255394.

33 Durante M, Reppingen N, Held KD. Immunologically augmented cancer treatment using modern radiotherapy. Trends Mol Med 2013;19:565-82.

34 Bos PD, Plitas G, Rudra D, et al. Transient regulatory T cell ablation deters oncogenedriven breast cancer and enhances radiotherapy. J Exp Med 2013;210:2435-66.

35 Gupta A, Probst HC, Vuong V, et al. Radiotherapy promotes tumor-specific effector CD8+ T cells via dendritic cell activation. J Immunol 2012;189:558-66.

36 Demaria S, Bhardwaj N, McBride WH, et al. Combining radiotherapy and immunotherapy: a revived partnership. Int J Radiat Oncol Biol Phys 2005;63:655-66.

37 De Wolf K, Vermaelen K, De Meerleer G, WK D, MG D, et al. The potential of radiotherapy to enhance the efficacy of renal cell carcinoma therapy. Oncoimmunology 2015;4:e1042198.

38 Zhou $Y$, Lin Z, Zhang $X$, et al. First-Line treatment for patients with advanced non-small cell lung carcinoma and high PD-L1 expression: pembrolizumab or pembrolizumab plus chemotherapy. J Immunother Cancer 2019;7:120.

39 Buttigliero C, Allis S, Tucci M, et al. Role of radiotherapy in improving activity of immune-modulating drugs in advanced renal cancer: biological rationale and clinical evidences. Cancer Treat Rev 2018;69:215-23.

40 Alissafi T, Hatzioannou A, Legaki Al, et al. Balancing cancer immunotherapy and immune-related adverse events: the emerging role of regulatory T cells. J Autoimmun 2019;104:102310.

41 Davies M, Duffield EA. Safety of checkpoint inhibitors for cancer treatment: strategies for patient monitoring and management of immune-mediated adverse events. Immunotargets Ther 2017;6:51-71

42 Brahmer JR, Lacchetti C, Schneider BJ, et al. Management of immune-related adverse events in patients treated with immune checkpoint inhibitor therapy: American Society of Clinical Oncology clinical practice guideline. J Clin Oncol 2018;36:1714-68.

43 Champiat S, Lambotte O, Barreau E, et al. Management of immune checkpoint blockade dysimmune toxicities: a collaborative position paper. Ann Oncol 2016;27:559-74.

44 Eggermont AMM, Chiarion-Sileni V, Grob J-J, et al. Prolonged survival in stage III melanoma with ipilimumab adjuvant therapy. N Engl J Med 2016;375:1845-55.

45 Martins F, Sofiya L, Sykiotis GP, et al. Adverse effects of immune-checkpoint inhibitors: epidemiology, management and surveillance. Nat Rev Clin Oncol 2019;16:563-80.

46 Buckley LM, Suarez-Almazor ME. Is immune checkpoint inhibitor treatment an option for patients with rheumatic diseases and cancer? Arthritis Rheumatol 2019;71:1971-3

47 Postow MA, Chesney J, Pavlick AC, et al. Nivolumab and ipilimumab versus ipilimumab in untreated melanoma. N Eng/ J Med 2015;372:2006-17.

48 Bomze D, Hasan Ali O, Bate A, et al. Association between immune-related adverse events during anti-PD-1 therapy and tumor mutational burden. JAMA Oncol 2019:5:1633-5.

49 Uemura M, Trinh VA, Haymaker C, et al. Selective inhibition of autoimmune exacerbation while preserving the anti-tumor clinical benefit using IL-6 blockade in a patient with advanced melanoma and Crohn's disease: a case report. J Hematol Oncol 2016;9:81

50 livanainen S, Koivunen JP. Early PD-1 therapy discontinuation in responding metastatic cancer patients. Oncology 2019;96:125-131.

51 Wang DY, Salem J-E, Cohen JV, et al. Fatal toxic effects associated with immune checkpoint inhibitors: a systematic review and meta-analysis. JAMA Oncol 2018:4:1721-8

52 Haanen JBAG, Carbonnel F, Robert C, et al. Management of toxicities from immunotherapy: ESMO clinical practice guidelines for diagnosis, treatment and follow-up. Ann Oncol 2018:29:iv264-6.

53 Perez BA, Kim S, Dilling TJ, et al. A prospective single arm phase I/II study: consolidative ipilimumab and nivolumab with thoracic radiotherapy after platinum based chemotherapy for patients with extensive-stage small cell lung cancer. Int J Radiat Oncol Biol Phys 2019:105:S36. 